\title{
Spin-polarized photoemission spectroscopy of the MgO/Fe interface on $\operatorname{GaAs}(100)$
}

\author{
F. Matthes ${ }^{\mathrm{a})}$ \\ Leibnitz-Institute for Solid State and Materials Research Dresden, Helmholtzstrasse 20, \\ 01069 Dresden, Germany \\ L.-N. Tong \\ Institute of Material Science and Engineer, Anhui University of Technology, Ma-An-Shan, \\ 243002 Anhui, China \\ C. M. Schneider \\ Institute of Solid State Research, Research Centre Jülich, 52425 Jülich, Germany
}

(Presented on 8 January 2004)

\begin{abstract}
We studied the electronic band states at the $\mathrm{Fe} / \mathrm{MgO}$ interface with spin-polarized photoemission using excitation energies between 35 and $60 \mathrm{eV}$. Epitaxial Fe films on $\mathrm{GaAs}(100)$ were covered with 0.5 , 1, and 1.5 ML thick MgO films. The photoemission spectra revealed a spin-dependent attenuation for photoelectrons related to direct transitions from initial bulk Fe bands having minority or majority spin character. ㅇ 2004 American Institute of Physics. [DOI: 10.1063/1.1669214]
\end{abstract}

Initiated by the possibility to grow epitaxial $\mathrm{Fe} / \mathrm{MgO} / \mathrm{Fe}$ trilayer structures on $\mathrm{GaAs}(100)$, this system became the system of choice for theoretical calculations of the tunnelling magneto-resistance (TMR) effect. ${ }^{1,2}$ In order to supply input for a correct modeling, it is of great importance to explore the properties of the $\mathrm{Fe} / \mathrm{MgO}$ interface. Only the knowledge of its structure and much more importantly of its electronic properties will allow one to get a deeper insight into the microscopic mechanisms ruling the TMR effect.

The interface structure is a crucial ingredient for the theoretical calculations. Up to now, the $\mathrm{Fe} / \mathrm{MgO}$ interface is assumed to form with an epitaxial match, so that the Fe[100] direction is parallel to the $\mathrm{MgO}[110]$ axis, requiring the $\mathrm{Fe}$ lattice to be rotated by $45^{\circ}$ with respect to the $\mathrm{MgO}$ lattice. ${ }^{3}$ Low-energy electron diffraction (LEED) studies suggested that the Fe atoms at the interface take positions directly on top of the $\mathrm{O}$ ions. ${ }^{4}$ This has been confirmed by total energy studies predicting that it is energetically more favorable to place the Fe atom on top of the neighboring oxygen instead of the $\mathrm{Mg}$ atom. ${ }^{5}$ In these calculations, performed by $\mathrm{Li}$ and Freeman, the charge transfer at the $\mathrm{Fe} / \mathrm{MgO}$ interface was determined to be less than $0.05 \mathrm{e} /$ atom. Furthermore, the magnetic moment of $1 \mathrm{ML} \mathrm{Fe}$ on $\mathrm{MgO}(100)$ was calculated to be $3.07 \mu_{B}$, which is remarkably close to that of a free standing Fe monolayer $\left(3.10 \mu_{B}\right)$. They observed only a minute magnetic moment of $0.03 \mu_{B}$ for the $\mathrm{O} 2 p$ states, introduced by hybridization with the $3 d$ states of the $\mathrm{Fe}$ overlayer at the vicinity of the $\Gamma$ point. Although these results suggest that the influence of the interface between $\mathrm{Fe}$ and $\mathrm{MgO}$ on the electronic structure is quite small and that both materials virtually behave the same as free surfaces, one has to be aware that this assumption can only be a first approximation for a realistic electronic structure at the inter-

a) Author to whom correspondence should be addressed; electronic mail: f.matthes@fz-juelich.de face. Up to now, theoretical values for the TMR-Mathon and Umerski predicted $1200 \%$ for a $\mathrm{MgO}$ barrier of $\sim 20$ atomic planes-are much larger than those measured experimentally. ${ }^{6}$ The reasons for this discrepancy are not yet clear. Mathon and Umerski observed that slight shifts of the on-site potential of one Fe electrode away from the nominal values can drastically influence the TMR ratio. Up to now, the values of this potential have been used to match the surface moment of the Fe interface layer ${ }^{2}$ or to correctly offset the $\mathrm{MgO}$ bands relative to those of $\mathrm{Fe}^{1}{ }^{1}$ Another reason may be that the existing models do not yet account for an interfacial roughness, structural disorder at the interfaces, or a possible substochiometric layer of $\mathrm{FeO}$ at the interface, as has been suggested by Meyerheim. ${ }^{7}$ Recently, Zhang and co-workers modeled the influence of an $\mathrm{FeO}$ interfacial layer between the first $\mathrm{Fe}$ electrode and the $\mathrm{MgO}$ barrier. They predicted that the tunneling conductance of the parallel configuration is reduced by 1 order of magnitude, leading to an overall reduced TMR value. ${ }^{8}$ Furthermore, they found a significant moment of $0.19 \mu_{B}$ on the $\mathrm{O}$ site of the $\mathrm{FeO}$ layer, which may be expected to alter the coupling of the electronic states of the Fe electrode into the $\mathrm{MgO}$.

It is thus mandatory to study the electronic structure at the interface and to clarify possible influences on it. It is worthwhile to mention that spin-integrated measurements may be insufficient to answer these questions, as due to the theoretically predicted small charge transfer between $\mathrm{Fe}$ and $\mathrm{MgO}$ as well as the predicted weak hybridization between $\mathrm{Fe}$ $3 d$ states and $\mathrm{O} 2 p$ states, the expected changes, e.g., shifts of the $\mathrm{Fe} 3 d$ bands may be too small to be resolved. As a first step, we therefore performed spin-resolved photoemission experiments and determined the spin-dependent changes of the photoelectron spectra as a function of the $\mathrm{MgO}$ barrier thickness.

The samples have been prepared under ultrahigh vacuum conditions. The base pressure was $2 \times 10^{-10}$ mbar. The 
GaAs(100) substrates have been cleaned with Ar ions until no carbon or oxygen contaminations were detectable by Auger electron spectroscopy (AES). Subsequently, the substrates have been annealed up to temperatures of $560^{\circ} \mathrm{C}$. The LEED pattern exhibited a $4 \times 6$ reconstruction, indicating a Ga-terminated surface. The Ga surface termination is believed to hinder a diffusion of As. On such prepared substrates $16 \mathrm{ML}$ thick Fe films were grown by electron-beam evaporation from $\mathrm{Fe}$ rod material of high purity $(99.995 \%)$. Subsequently performed AES studies revealed no carbon or oxygen contaminations of the Fe surface. LEED investigations proved the bcc structure of the Fe film. In situ magnetooptical Kerr effect measurements confirmed a ferromagnetic ordering at room temperature with the easy axis oriented along the [110] direction. ${ }^{9}$

The spin- and angle-resolved photoemission experiments were performed at the undulator beam line U-125-1 PGM (BESSY). The beam line offers linear polarized light with the electric field vector lying in the plane defined by the $k$ vector of the incident beam and the $k$ vector of the detected photoelectrons. The incident light made an angle of $45^{\circ}$ with the surface normal. In this geometry we have components of the electric field vector in-plane along the [110] direction and perpendicular to the sample surface. The photoelectrons were detected along the surface normal. Following the dipole selection rules such a geometry allows direct transitions from initial states with $\Delta_{1}$ and $\Delta_{5}$ spatial symmetry into final states with $\Delta_{1}$ spatial symmetry. ${ }^{10}$ The energy of the photoelectrons was determined using a cylindrical mirror type analyzer with integrated LEED spin-polarization detector $\left(\right.$ CSA200-SPLEED combination $\left.{ }^{11}\right)$. The overall energy resolution defined by the spectral resolution of the beam line, the angular acceptance of the entrance electron optics $\left(6^{\circ}\right)$, pass energy of the analyzer, and slit width was nominally 200 $\mathrm{meV}$. The spin polarization components of the photoelectrons perpendicular to the sample surface and in-plane along the [110] direction were determined simultaneously.

For the analysis of our spin-resolved photoemission data we first subtracted a background intensity originating from secondary electrons generated by higher order light. In the next step, we calculated the spin polarization, using two subsequently measured spectra: the first one having the magnetization vector aligned along the [110] direction and the second one with the magnetization direction reversed by $180^{\circ}$. This procedure eliminates apparatus-related asymmetries. Finally, based on the determined spin polarization we recalculated the partial intensities of the minority and majority spin components. Figure 1 depicts a set of spin-resolved photoemission spectra from a $16 \mathrm{ML}$ thick Fe film on GaAs(100) measured at a photon energy of $35 \mathrm{eV}$. To identify possible direct transitions from bulk initial states involved in the photoemission process, we performed a spin polarized relativistic Korringa-Kohn-Rostocker band structure calculation for bulk bcc $\mathrm{Fe}(100) .{ }^{12}$ Comparing Figs. 1 and 2 we can identify three direct transitions presumably originating from the initial state bands $\Delta_{6}^{1 \downarrow}, \Delta_{6,7}^{5 \downarrow}, \Delta_{6}^{1 \uparrow}$. Our result is consistent with the interpretation of Turner and co-workers. ${ }^{13}$ As we have no access yet to one-step model photoemission calculations for this system, we will stay within the three-step model of pho-

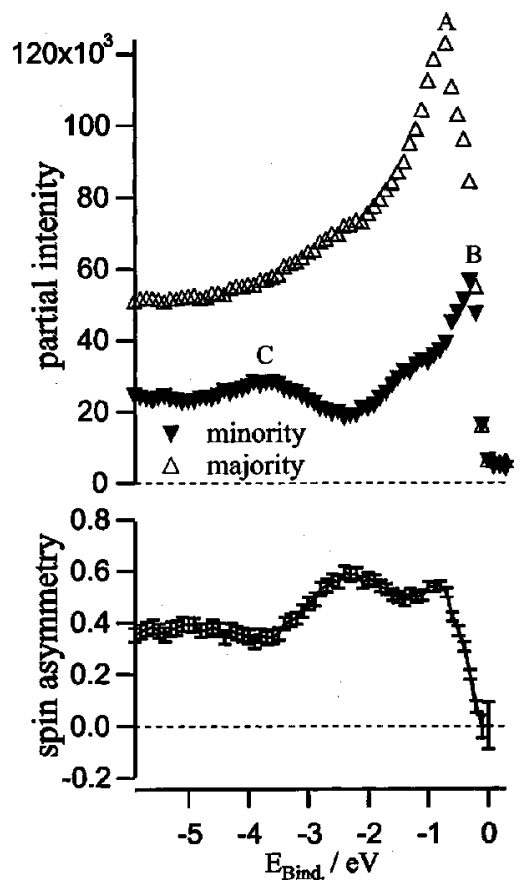

FIG. 1. Spin-resolved photoemission of $16 \mathrm{ML} \mathrm{Fe}$ on GaAs(100). Labeled are direct transitions to final states with $\Delta_{6}^{1}$ symmetry from initial bulk Fe band (A) $=\Delta_{6}^{1 \uparrow}$, (B) $\Delta_{6,7}^{5 \downarrow}$, and (C) $\Delta_{6}^{1 \downarrow}$.

toemission for our further argumentation. Within this model main features in the photoemission spectra may be related to direct transitions. We neglect the influence of photoemission emerging from surface states, surface resonances, or via indirect transitions. In the case of $\mathrm{Fe}(100)$, these transitions are dominant for excitation energies between approximately 15

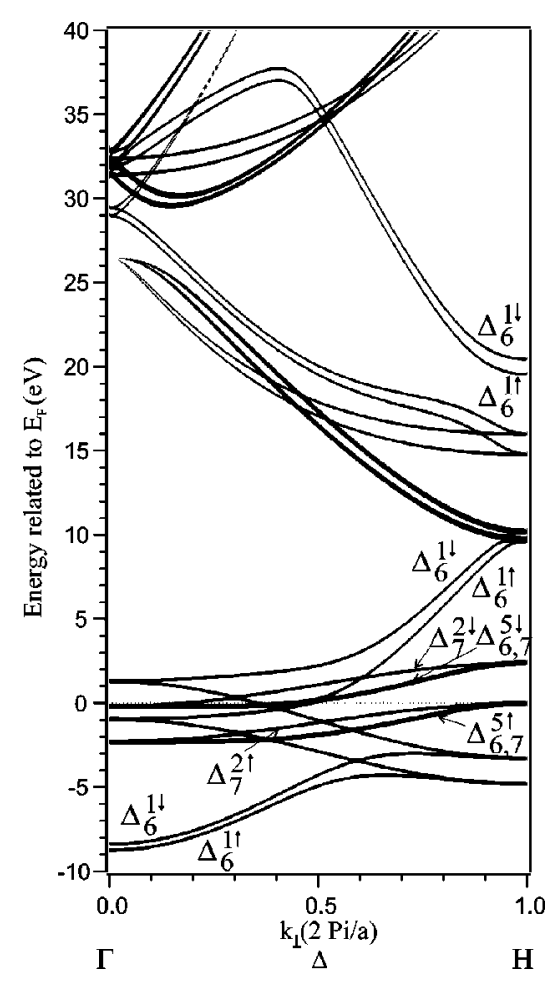

FIG. 2. Spin polarized relativistic Korringa-Kohn-Rostocker band structure calculation for bulk bcc Fe(100) (see Ref. 9). 

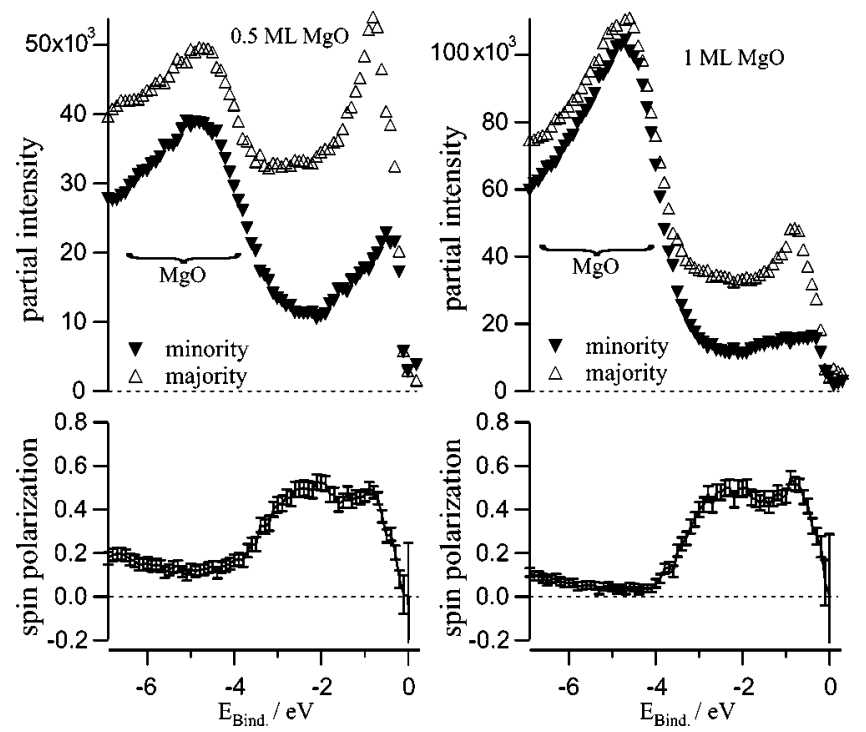

FIG. 3. Spin-resolved photoemission of nominal $0.5 \mathrm{ML}$ thick $\mathrm{MgO}$ (left side) and $1 \mathrm{ML}$ thick $\mathrm{MgO}$ (right side) on $16 \mathrm{ML} \mathrm{Fe} / \mathrm{GaAs}(100)$ measured at a photon energy of $35 \mathrm{eV}$.

and $30 \mathrm{eV} \cdot{ }^{13,14}$ This is caused by a band gap in the final band structure $\left(\Delta_{6}^{1 \downarrow, \uparrow}\right.$ states $)$ preventing direct transitions. To assure that the photoemission spectra are governed by direct transitions, we restricted our evaluation to excitation energies higher than $30 \mathrm{eV}$.

In the next step we have grown $\mathrm{MgO}$ films onto the 16 ML thick Fe film by electron-beam evaporation from a $\mathrm{Mg}$ rod and simultaneous oxidation in an oxygen partial pressure of $1 \times 10^{-8}$ mbar, whereby the oxygen was guided through a nozzle directly to the sample surface. The success of the oxidation procedure was verified by comparing the Auger electron spectrum of the oxidized $\mathrm{Mg}$ film with that of a $\mathrm{MgO}$ crystal. Figure 3 depicts the spin-resolved data for a nominal coverage of 0.5 and $1 \mathrm{ML} \mathrm{MgO}$. With increasing thickness of the $\mathrm{MgO}$ film we observe two features: the spectral intensity originating from the valence band of $\mathrm{MgO}^{15}$ increases while the intensity of direct transitions from the $\mathrm{Fe}$ bulk-related states $\Delta_{6}^{1 \uparrow}$ and $\Delta_{6,7}^{5 \downarrow}$ is suppressed. As a surprising finding, our spin-resolved measurements reveal that the attenuation for the direct transition from the $\Delta_{6,7}^{5 \downarrow}$ band is stronger than the one from the $\Delta_{6}^{1 \uparrow}$ band. This behavior cannot be simply related to differences in the spatial symmetry of the photoelectron wave functions, as the final states for both transitions have $\Delta_{6}^{1}$ symmetry. The two direct Fe-related transitions are also not overlapping with any $\mathrm{MgO}$-related transitions, as bulk $\mathrm{MgO}$ is an insulator with a band gap of $7.8 \mathrm{eV}^{16}$ Theoretical calculations for bulk $\mathrm{MgO}$ placed the Fermi energy $3.5 \mathrm{eV}$ above the valence band edge of $\mathrm{MgO} .^{5}$ For ultrathin films of $\mathrm{MgO}$ Klaua and co-workers ${ }^{16}$ measured a thickness dependent tunneling barrier with a minimum value of $2.6 \mathrm{eV}$ for $2 \mathrm{ML}$. Assuming, as also shown by theoretical calculations, ${ }^{6}$ that the Fermi level is located ap- proximately in the middle of the gap, we can thus exclude any spectral contributions from $\mathrm{MgO}$ in the region of $2 \mathrm{eV}$ below the Fermi level.

There are several possible mechanisms, which may be important to explain our observation. First, a possible formation of interface and half-space states and the scattering of the excited electrons at the interfaces and within the $\mathrm{MgO}$ film, which can be taken into account only by a proper onestep photoemission calculation. The second mechanism is based on the fact that the final states of $\mathrm{Fe}$ are exchange split. The $\Delta_{6}^{1 \downarrow}$ and $\Delta_{6}^{1 \uparrow}$ are shifted in energy and may thus couple to different final states in the $\mathrm{MgO}$ film, or to the same final state at different $k_{\perp}$ values. Thus, different coupling coefficients at the interface may be involved, causing a spin dependence. The third mechanism relates to a possible $\mathrm{FeO}$ layer at the interface. In this case, Zhang and co-workers ${ }^{8}$ predicted a charge transfer and an induced magnetic moment of $0.19 \mu_{B}$ at the $\mathrm{O}$ atoms of the interfacial $\mathrm{FeO}$. Thus, one can assume that the spin-split density of states at the $\mathrm{Fe} / \mathrm{FeO} /$ $\mathrm{MgO}$ interface will be altered, giving rise to an additional spin-dependent contribution. Which of these mechanisms is the dominant one cannot be decided on the basis of the present experimental data. Further investigations backed by theoretical one-step photoemission calculations will be necessary to elucidate the behavior of the $\mathrm{Fe} / \mathrm{MgO}$ interface.

In conclusion, we have observed a spin-dependent attenuation for direct transitions related to $\mathrm{Fe}$ bulk initial states as a function of the $\mathrm{MgO}$ thickness. As this attenuation was measured also at photon energies of 40 and $60 \mathrm{eV}$ (not shown here), we consider it a general property of the interface. To clarify its origin, theoretical calculations of the photoemission and maybe further experimental work have to be performed.

${ }^{1}$ W. H. Butler et al., Phys. Rev. B 63, 054416 (2001).

${ }^{2}$ J. Mathon and A. Umerski, Phys. Rev. B 63, 220403(R) (2001).

${ }^{3}$ T. Kanaji, K. Asano, and S. Nagata, Vacuum 23, 55 (1973).

${ }^{4}$ T. Urano and T. Kanaji, Thin Solid Films 32, 217 (1976).

${ }^{5}$ C. Li and A. J. Freeman, Phys. Rev. B 43, 780 (1991).

${ }^{6}$ E. Popova et al., Appl. Phys. Lett. 81, 1035 (2002); M. Bowen et al., ibid. 79, 1655 (2001).

${ }^{7}$ H. L. Meyerheim et al., Phys. Rev. B 65, 144433 (2002); Phys. Rev. Lett. 87, 076102 (2001).

${ }^{8}$ X.-G. Zhang et al., Phys. Rev. B 68, 092402 (2003).

${ }^{9} \mathrm{~A}$ detailed description will be published together with the structure and magnetic properties of Co films grown on $\mathrm{Fe} / \mathrm{GaAs}(100)$.

${ }^{10}$ W. Eberhardt and F. J. Himpsel, Phys. Rev. B 21, 5572 (1980).

${ }^{11}$ Focus GmbH, Germany.

${ }^{12} \mathrm{H}$. Ebert et al., The Munich SPR-KKR package, version 2.1, 〈http:// olymp.cup.uni-muenchen.de/ak/ebert/SPRKKR $\rangle$; H. Ebert, Fully Relativistic Band Structure Calculations for Magnetic Solids-Formalism and Application, in Electronic Structure and Physical Properties of Solids, edited by H. Dreyssé, Lecture Notes in Physics, Vol. 535 (Springer, Berlin, 2000), p. 191.

${ }^{13}$ A. M. Turner, A. W. Donoho, and J. L. Erskine, Phys. Rev. B 29, 2986 (1984); A. M. Turner and J. L. Erskine, ibid. 30, 6675 (1984).

${ }^{14}$ E. Kisker, K. Schröder, W. Gudat, and M. Campagna, Phys. Rev. B 31, 329 (1985).

${ }^{15}$ M. Grass, J. Braun, and G. Borstel, Surf. Sci. 334, 215 (1995).

${ }^{16}$ M. Klaua et al., Phys. Rev. B 64, 134411 (2001). 